\title{
A Spherically Symmetric Model for the Tumor Growth
}

\author{
Saeed M. Ali, Ashfaque H. Bokhari, M. Yousuf, and F. D. Zaman \\ Department of Mathematics and Statistics, King Fahd University of Petroleum \& Minerals, Dhahran 31261, Saudi Arabia \\ Correspondence should be addressed to F. D. Zaman; fzaman@kfupm.edu.sa
}

Received 25 May 2013; Accepted 19 December 2013; Published 19 January 2014

Academic Editor: Renat Zhdanov

Copyright (C) 2014 Saeed M. Ali et al. This is an open access article distributed under the Creative Commons Attribution License, which permits unrestricted use, distribution, and reproduction in any medium, provided the original work is properly cited.

\begin{abstract}
The nonlinear tumor equation in spherical coordinates assuming that both the diffusivity and the killing rate are functions of concentration of tumor cell is studied. A complete classification with regard to the diffusivity and net killing rate is obtained using Lie symmetry analysis. The reduction of the nonlinear governing equation is carried out in some interesting cases and exact solutions are obtained.
\end{abstract}

\section{Introduction}

The tumor growth has been usually modeled as a reactiondiffusion process in the literature. Jones et al. [1] have given a simple tumor model based upon this idea. A model describing the growth of the tumor in brain taking into account diffusion or motility as well as proliferation of tumor cells has been developed in a series of papers [2,3]. In continuation of this approach, Tracqui et al. [4] suggest a model which takes into account treatment and thus killing rate of tumor cells along with the above factors. The governing equation in this case is

$$
u_{t}=D \nabla^{2} u+P u-k u
$$

where $u$ is the concentration of tumor cells, $D$ is the diffusion coefficient, $P$ is the proliferation rate, and $k$ is the killing rate. Assuming complete radial summery, Moyo and Leach [3] have studied this model with $K(x, t)=P-k$ being variable. The resulting governing equation reduces to the simple form

$$
u_{t}=u_{x x}-K u \text {, }
$$

where $u(x, t)=r u(r, t)$. They have performed Lie symmetry analysis and presented some exact solutions based upon this approach. Consequently, Bokhari et al. [5] used Lie symmetry analysis to obtain a number of invariant reductions and exact solutions in the case of killing rate $K(u)$ being function of $u$. The present study is based upon the fact that the diffusivity is not necessarily a constant and may depend upon the concentration of tumor cells. Moreover, the net killing rate $K$ is also taken to be $u$-dependent. This introduces nonlinearity in the governing equation. Keeping these assumptions in mind (1) becomes

$$
u_{t}=\nabla \cdot(D(u) \nabla u)-K(u) u,
$$

which in spherical coordinates and with radial symmetry assumption becomes

$$
\frac{1}{x^{2}} \frac{\partial}{\partial x}\left(x^{2} D(u) u_{x}\right)-K(u) u=u_{t},
$$

where $D(u)$ is the diffusivity of the medium and $K(u)$ is the net killing rate. We present a classification of the functions $D(u)$ and $K(u)$ using Lie symmetry analysis. The Lie symmetry approach, first proposed by Lie [6], has been used to classify nonlinear differential equations, find appropriate similarity transformations, and find exact solutions. One may refer to [7-10] for a good account of this method. Some recent studies in nonlinear diffusion equations using this approach can be found in $[1,6]$.

\section{Symmetry Analysis of the Tumor Equation}

In this section, we perform the symmetry analysis of (4). To this end, we use the Lie symmetry method [10] which is based upon finding Lie point symmetries of the PDEs that leave them invariant. In order to find the Lie symmetry generators of (4) and obtain closed-form solutions for all $K(u)$, we 
consider the one-parameter Lie point transformation that leaves (4) invariant. These transformation formulae [7, 9] are given as follows:

$$
\tilde{x}^{i}=x^{i}+\epsilon \xi^{i}(x, y, t, u)+O\left(\epsilon^{2}\right), \quad i=1, \ldots, 4,
$$

where $\xi^{i}=\left.\left(\partial \tilde{x}^{i} / \partial \epsilon\right)\right|_{\epsilon=0}$ defines the symmetry generator [9] associated with (2) given by

$$
V=\xi \frac{\partial}{\partial x}+\eta \frac{\partial}{\partial y}+\tau \frac{\partial}{\partial t}+\phi \frac{\partial}{\partial u} .
$$

Requiring invariance of (4) with respect to the prolonged symmetry generator,

$$
\mathbf{V}^{(2)}=V+\sum_{I=0}^{2} \phi^{J} \frac{\partial}{\partial u_{J}}+\sum_{I, J=0}^{3} \phi^{I J} \frac{\partial}{\partial u_{I J}}, \quad I, J=0,1,
$$

with 0 representing $t$ and 1 representing $x$,

$$
\begin{aligned}
\xi\left(D(u) u_{x x}+D_{u} u_{x}^{2}-k(u) u-u_{t}\right) \\
+\phi\left(x D_{u} u_{x x}+x D_{u u} u_{x}^{2}+2 D_{u} u_{x}-x k(u)-x u k_{u}\right) \\
+\phi^{x}\left(2 x D_{u} u_{x}+2 D(u)\right)-x \phi^{t}+x D(u) \phi^{x x}=0
\end{aligned}
$$

and the coefficients $\phi^{J}$ and $\phi^{J K}$, of the derivatives with respect to dependent variables in (8) are to be evaluated using the expressions:

$$
\begin{gathered}
\phi^{J}=D_{i}\left(\phi-\xi^{j} u_{j i}\right)+\xi^{j} u_{j, i}, \\
\phi^{J K}=D_{i} D_{j}\left(\phi-\xi^{j} u_{j i}\right)+\xi^{k} u_{k, i j} .
\end{gathered}
$$

Using (9) into (8) and comparing terms involving derivatives of the dependent variable $u$, we obtain the following system of differential equations:

$$
\begin{gathered}
\xi_{u}=0=\tau_{x}=\tau_{u}=\phi_{u u}, \\
D_{u} \phi-2 D \xi_{x}+D \tau_{t}=0, \\
-x K \phi-x u K_{u} \phi+2 D \phi_{x}-x \phi_{t}+x u K \phi_{u} \\
-x u K \tau_{t}+x D \phi_{x x}=0, \\
-2 D \xi+2 x D_{u} \phi-2 x D \xi_{x}+2 x D \tau_{t}+x^{2} \xi_{t} \\
+2 D x^{2} \phi_{x u}-D x^{2} \xi_{x x}=0 .
\end{gathered}
$$

To determine the unknown functions $\xi, \tau$, and $\phi$, we solve the above coupled system of differential equations by first considering (11). Differentiating this equation twice with respect to $u$ leads to the following expression:

$$
\phi_{u u}=\left(\frac{D}{D_{u}}\right)_{u u}\left(2 \xi_{x}-\tau_{t}\right) .
$$

Using (10) into (14) reduces to

$$
\left(\frac{D}{D_{u}}\right)_{u u}\left(2 \xi_{x}-\tau_{t}\right)=0 .
$$

We proceed from the above equation to obtain a complete classification of both $D$ and $K$ as shown in the next section.

\section{Classification}

In order to perform a complete classification of solution of (4), we notice that the following three cases arise from (15)

$$
\begin{aligned}
& \text { (I) } 2 \xi_{x}-\tau_{t}=0, \\
& \text { (II) }\left(D / D_{u}\right)_{u u}=0, \\
& \text { (III) } 2 \xi_{x}-\tau_{t}=0=\left(D / D_{u}\right)_{u u} \text {. }
\end{aligned}
$$

For obtaining a complete classification, we consider all the three cases one by one. Since procedure of classification in all the three cases is similar, we present a complete analysis in the first case and briefly state the results in the remaining cases. To begin the classification, we proceed as follows.

3.1. Case $I\left(2 \xi_{x}-\tau_{t}=0\right)$. In this case the system of determining equations given by (10)-(13) becomes

$$
\begin{gathered}
\xi_{u}=0=\tau_{x}=\tau_{u}=\phi, \\
2 \xi_{x}=\tau_{t}, \\
-x u K \tau_{t}=0, \\
-2 D \xi+x D \tau_{t}+x^{2} \xi_{t}=0 .
\end{gathered}
$$

From (18), three subcases arise:
(a) $\tau_{t}=0$,
(b) $K(u)=0$,
(c) $\tau_{t}=0=K(u)$.

We first consider (a).

3.1.1. Subcase (a) $\left(\tau_{t}=0\right)$. Using these conditions arising in this case, we deduce that

$$
\xi_{x}=0 \Longrightarrow \xi=\xi(t),
$$

and (19) becomes

$$
-2 D \xi+x^{2} \xi_{t}=0
$$

Note that (21) is a separable DE and can be easily solved to find $\xi$ given by $\xi=c_{2} \exp \left(-2 D x^{-2} t\right)$.

But $\xi$ depend only on $t$, so that two possibilities arise from expression of $\xi$ :

(a.1) $c_{2}=0$ and $D \neq 0$,

(a.2) $D=0$ and $c_{2} \neq 0$.

Subsubcase (a.1). This subcase leads to the fact that $D(u)$ and $K(u)$ are arbitrary and the infinitesimals are

$$
\xi=0, \quad \tau=c_{1}, \quad \phi=0 .
$$

Only one symmetry generator is associated with above infinitesimals which is $\mathbf{X}=(\partial / \partial t)$.

Subsubcase (a.2). As a result of this subcase, $D(u)=0$ and $K(u)$ are arbitrary and the infinitesimals are

$$
\xi=c_{2}, \quad \tau=c_{1}, \quad \phi=0 .
$$


TABLE 1: Commutator table of the tumor equation.

\begin{tabular}{lcc}
\hline$\left[\mathrm{X}_{\mathrm{i}}, \mathrm{X}_{\mathrm{j}}\right]$ & $\mathrm{X}_{1}$ & $\mathrm{X}_{2}$ \\
\hline $\mathrm{X}_{1}$ & 0 & $-2 \mathrm{X}_{2}$ \\
$\mathrm{X}_{2}$ & $2 \mathrm{X}_{2}$ & 0 \\
\hline
\end{tabular}

In this subcase, we have two generators which are

$$
\mathbf{X}_{1}=\frac{\partial}{\partial x}, \quad \mathbf{X}_{2}=\frac{\partial}{\partial t}
$$

3.1.2. Subcase $(b)(K(u)=0)$. Using these conditions arising in this case into (13), it becomes

$$
-2 D \xi+2 x D \xi_{x}+x^{2} \xi_{t}+3 D x^{2} \xi_{x x}=0
$$

Differentiating (25) with respect to $u$, we get

$$
3 x^{2} \xi_{x x}+2 x \xi_{x}-2 \xi=0 .
$$

The above equation is a Cauchy Euler differential equation, its solution given by

$$
\xi=A(t) x+B(t) x^{-(2 / 3)} .
$$

To require consistency of $\xi$ found above, we use (27) into (25). This suggests that (25) is satisfied when the following differential constraint is met:

$$
A_{t} x^{3}+B_{t} x^{4 / 3}=0
$$

Differentiating (28) four times with respect to $x$, we get $B(t)=$ $c_{2}$ and hence $A(t)=c_{1}$. Therefore,

$$
\xi=c_{1} x+c_{2} x^{-(2 / 3)}
$$

Using (29) into (17), we conclude that

$$
\tau=2 c_{1} t-\frac{4}{3} c_{2} x^{-(5 / 3)}+c_{3}
$$

From (16) and (30), we infer that $c_{2}=0$ and consequently the general expressions of $\xi, \tau, \phi, D$, and $K$ are

$$
\begin{gathered}
\xi=c_{1} x, \quad \tau=2 c_{1} t+c_{3}, \quad \phi=0 . \\
K(u)=0, \quad D(u) \text { is arbitrary. }
\end{gathered}
$$

The two symmetry generators associated with above infinitesimals are given by

$$
X_{1}=x \frac{\partial}{\partial x}+2 t \frac{\partial}{\partial t}, \quad X_{2}=\frac{\partial}{\partial t} .
$$

The commutation relation for each of the above symmetry generators is listed in Table 1.

3.1.3. Subcase (c) $\left(\tau_{t}=0=K(u)\right)$. Following the procedure adopted in earlier cases, we obtain the same symmetry generators in cases (a) and (b).
3.2. Case $I I\left(\left(D / D_{u}\right)_{u u}=0\right)$. Solving equation $\left(D / D_{u}\right)_{u u}=0$ for $D(u)$ instantly yields

$$
D(u)=(b u)^{1 / b},
$$

where $b$ is a constant. Using (33) into (11), we obtain

$$
\phi=(b u)\left(2 \xi_{x}-\tau_{t}\right) .
$$

Using (34) and (33) into (13), we obtain a differential relation in the $\xi$ given by

$$
\begin{array}{r}
-2(b u)^{1 / b} \xi+2 x(b u)^{1 / b} \xi_{x}+x^{2} \xi_{t} \\
+x^{2}(4 b-1)(b u)^{1 / b} \xi_{x x}=0 .
\end{array}
$$

Differentiating (35) with respect to $u$, we obtain

$$
-2 \xi+2 x \xi_{x}+x^{2}(4 b-1) \xi_{x x}=0 .
$$

From (36) two cases arise:
(a) $b \neq 1 / 4$,
(b) $b=1 / 4$.

3.2.1. Subcase $(a)(b \neq 1 / 4)$. This subcase gives that

$$
\xi=A(t) x+B(t) x^{-2 / m_{1}}, \quad \text { where } m_{1}=4 b-1 .
$$

Using (37) into (35) yields

$$
A_{t} x^{3}+B_{t} x^{-\left(2 / m_{1}\right)+2}=0 .
$$

Differentiating (38) with respect to $x 4$ times, we conclude that $B(t)=c_{2}$ and hence $A(t)=c_{1}$. Therefore,

$$
\xi=c_{1} x+c_{2} x^{-2 / m_{1}}, \quad \text { where } m_{1}=4 b-1 .
$$

As a result of (39), we obtain

$$
\phi=b u\left(2 c_{1}-\frac{4}{m_{1}} c_{2} x^{-\left(2 / m_{1}\right)-\tau_{t}}\right) .
$$

Using (39) and (40) into (12), we obtain

$$
\begin{aligned}
& -b u^{2} K_{u}\left(2 c_{1}-\frac{4}{m_{1}} c_{2} x^{-\left(2 / m_{1}\right)-1}-\tau_{t}\right)+b u \tau_{t t}-u K \tau_{t} \\
& -\frac{8}{m_{1}} c_{2}(b u)^{(1 / b)+1}\left(-\frac{2}{m_{1}}-1\right) x^{-\left(2 / m_{1}\right)-3} \\
& -\frac{4}{m_{1}} c_{2}(b u)^{(1 / b)+1}\left(-\frac{2}{m_{1}-1}\right)\left(-\frac{2}{m_{1}-2}\right) x^{-\left(2 / m_{1}\right)-3}=0 .
\end{aligned}
$$

Differentiating (41) with respect to $x$ yields

$$
\begin{aligned}
-b u^{2} & K_{u} \frac{4}{m_{1}}\left(\frac{-2}{m_{1}}-1\right) c_{2} x^{\left(-2 / m_{1}\right)-2}-\frac{8}{m_{1}} c_{2}(b u)^{(1 / b)+1} \\
& \times\left(-\frac{2}{m_{1}}-1\right)\left(-\frac{2}{m_{1}}-3\right) x^{-\left(2 / m_{1}\right)-4}-\frac{4}{m_{1}} c_{2}(b u)^{(1 / b)+1} \\
& \times\left(-\frac{2}{m_{1}}-1\right)\left(-\frac{2}{m_{1}}-1\right)\left(-\frac{2}{m_{1}}-3\right) x^{-\left(2 / m_{1}\right)-4}=0 .
\end{aligned}
$$


The above equation holds if $c_{2}=0$, and substituting this value into (41) gives

$$
-b u K_{u}\left(2 c_{1}-\tau_{t}\right)+b \tau_{t t}-K \tau_{t}=0 .
$$

Differentiating (43) with respect to $t$

$$
-b u K_{u}-K=\frac{-b \tau_{t t t}}{\tau_{t t}}
$$

The equality in (44) holds if

$$
-b u K_{u}-K=\frac{-b \tau_{t t t}}{\tau_{t t}}=\gamma
$$

where $\gamma$ is a constant.

In accordance with (45), we conclude that

$$
\begin{gathered}
\tau(t)=\frac{c_{3} b^{2}}{\gamma^{2}} \exp \left(\frac{\gamma}{b} t\right)+c_{4} t+c_{5}, \\
K(u)=\gamma+\lambda u^{1 / b} .
\end{gathered}
$$

Using (46) into (43) yields

$$
-2 c_{1} \lambda u^{1 / b}-\gamma c_{4}=0 .
$$

Differentiating (47) with respect to $u$ leads to

$$
-2 c_{1} \frac{1}{b} \lambda u^{(1 / b)-1}=0
$$

From (48) three cases arise, namely,

$$
\begin{aligned}
& \text { (a.1) } c_{1}=0 \text {, and } \lambda \neq 0, \\
& \text { (a.2) } c_{1} \neq 0 \text { and } \lambda=0, \\
& \text { (a.3) } c_{1}=0 \text { and } \lambda=0 .
\end{aligned}
$$

Subsubcase $(a .1)\left(D(u)=(b u)^{1 / b}\right.$ and $\left.K(u)=\gamma+\lambda u^{1 / b}\right)$. Using these conditions arising in this case into (47), we obtain $c_{4}=0$ and hence the infinitesimals are determined

$$
\begin{gathered}
\xi=0, \quad \tau=\frac{c_{3} b}{\gamma} \exp \left(\frac{\gamma}{b} t\right)+c_{5}, \\
\phi=-c_{3} b u \exp \left(\frac{\gamma}{b} t\right) .
\end{gathered}
$$

The two symmetry generators associated with the above infinitesimals are given by

$$
\begin{gathered}
\mathbf{X}_{1}=\frac{b}{\gamma} \exp \left(\frac{\gamma}{b} t\right) \frac{\partial}{\partial t}-b u \exp \left(\frac{\gamma}{b} t\right) \frac{\partial}{\partial u}, \\
\mathbf{X}_{2}=\frac{\partial}{\partial t} .
\end{gathered}
$$

The commutation relation for these generators is given in Table 2 .
TABLE 2: Commutator table of the tumor equation.

\begin{tabular}{lcc}
\hline$\left[\mathbf{X}_{\mathbf{i}}, \mathbf{X}_{\mathrm{j}}\right]$ & $\mathbf{X}_{1}$ & $\mathbf{X}_{2}$ \\
\hline $\mathbf{X}_{1}$ & 0 & $-\frac{\gamma}{b} \mathbf{X}_{1}$ \\
$\mathbf{X}_{2}$ & $\frac{\gamma}{b} \mathbf{X}_{1}$ & 0 \\
\hline
\end{tabular}

TABLE 3: Commutator table of the tumor equation.

\begin{tabular}{lccc}
\hline$\left[\mathbf{X}_{\mathbf{i}}, \mathbf{X}_{\mathbf{j}}\right]$ & $\mathbf{X}_{\mathbf{1}}$ & $\mathbf{X}_{2}$ & $\mathbf{X}_{\mathbf{3}}$ \\
\hline $\mathbf{X}_{1}$ & 0 & 0 & $-\frac{\gamma}{b} \mathbf{X}_{\mathbf{1}}$ \\
$X_{2}$ & 0 & 0 & 0 \\
$X_{3}$ & $\frac{\gamma}{b} \mathbf{X}_{1}$ & 0 & 0 \\
\hline
\end{tabular}

Subsubcase (a.2) $\left(D(u)=(b u)^{1 / b}\right.$ and $\left.K(u)=\gamma\right)$. Similarly, using these conditions arising in this case into (47), we obtain $c_{4}=0$ and hence the infinitesimals are determined

$$
\begin{gathered}
\xi=c_{1} x, \quad \tau=\frac{c_{3} b}{\gamma} \exp \left(\frac{\gamma}{b} t\right)+c_{5}, \\
\phi=b u\left(2 c_{1}-c_{3} \exp \left(\frac{\gamma}{b} t\right)\right) .
\end{gathered}
$$

The three symmetry generators associated with the above infinitesimals are given by

$$
\begin{gathered}
\mathbf{X}_{1}=\frac{b}{\gamma} \exp \left(\frac{\gamma}{b} t\right) \frac{\partial}{\partial t}-b u \exp \left(\frac{\gamma}{b} t\right) \frac{\partial}{\partial u}, \\
\mathbf{X}_{2}=x \frac{\partial}{\partial x}+2 b u \frac{\partial}{\partial u}, \quad \mathbf{X}_{3}=\frac{\partial}{\partial t} .
\end{gathered}
$$

The commutation relation for these generators is given in Table 3.

Subsubcase $(a .3)\left(D(u)=(b u)^{1 / b}\right.$ and $\left.K(u)=\gamma\right)$. using these conditions arising in this case into (47), we obtain $c_{4}=0$ and hence the infinitesimals are

$$
\begin{gathered}
\xi=0, \quad \tau=\frac{c_{3} b}{\gamma} \exp \left(\frac{\gamma}{b} t\right)+c_{5}, \\
\phi=-c_{3} b u \exp \left(\frac{\gamma}{b} t\right) .
\end{gathered}
$$

The two symmetry generators associated with the above infinitesimals are given by

$$
\mathbf{X}_{1}=\frac{b}{\gamma} \exp \left(\frac{\gamma}{b} t\right) \frac{\partial}{\partial t}-b u \exp \left(\frac{\gamma}{b} t\right) \frac{\partial}{\partial u}, \quad \mathbf{X}_{2}=\frac{\partial}{\partial t} .
$$

The commutation relation for these generators is given in Table 4.

3.2.2. Subcase (b) $(b=1 / 4)$. In accordance with this subcase, (35) becomes

$$
2 x \xi_{x}-2 \xi=0 ; \quad \text { then } \xi=x \beta(t) .
$$


TABLE 4: Commutator table of the tumor equation.

\begin{tabular}{lcc}
\hline$\left[\mathbf{X}_{\mathrm{i}}, \mathbf{X}_{\mathrm{j}}\right]$ & $\mathbf{X}_{1}$ & $\mathbf{X}_{2}$ \\
\hline $\mathbf{X}_{1}$ & 0 & $-\frac{\gamma}{b} \mathbf{X}_{1}$ \\
$\mathbf{X}_{2}$ & $\frac{\gamma}{b} \mathbf{X}_{1}$ & 0 \\
\hline
\end{tabular}

Using (33) into (35) gives $\beta=c_{1}$ and hence

$$
\xi=c_{1} x, \quad \phi=\frac{1}{4} u\left(2 c_{1}-\tau_{t}\right) .
$$

Using (56) into (12) yields

$$
-\frac{1}{4} u K_{u}\left(2 c_{1}-\tau_{t}\right)+\frac{1}{4} \tau_{t t}-K \tau_{t}=0 .
$$

Differentiating (57) with respect to $t$

$$
-\frac{1}{4} u K_{u}-K=\frac{-(1 / 4) \tau_{t t t}}{\tau_{t t}}
$$

The equality in (58) holds if

$$
-b u K_{u}-K=\frac{-(1 / 4) \tau_{t t t}}{\tau_{t t}}=\gamma, \quad \text { where } \gamma \text { is a constant. }
$$

In accordance with (59), we have

$$
\tau(t)=\frac{c_{3}}{16 \gamma^{2}} \exp (4 \gamma t)+c_{4} t+c_{5}, \quad K(u)=\gamma+\lambda u^{4} .
$$

Using (60) into (57) yields

$$
-2 c_{1} \lambda u^{4}-\gamma c_{4}=0 .
$$

Differentiating (61) with respect to $u$ leads to

$$
-8 c_{1} \lambda u^{3}=0
$$

From (62) three cases arise:

$$
\begin{aligned}
& \text { (b.1) } c_{1}=0 \text {, and } \lambda \neq 0, \\
& \text { (b.2) } c_{1} \neq 0 \text { and } \lambda=0, \\
& \text { (b.3) } c_{1}=0 \text { and } \lambda=0 .
\end{aligned}
$$

Subsubcase (b.1) $\left(D(u)=((1 / 4) u)^{4}\right.$ and $\left.K(u)=\gamma+\lambda u^{4}\right)$. In accordance with this subsubcase and (61), we obtain $c_{4}=0$ and hence the infinitesimals are determined as

$$
\begin{gathered}
\xi=0, \quad \tau=\frac{c_{3}}{4 \gamma} \exp (4 \gamma t)+c_{5}, \\
\phi=-\frac{1}{4} c_{3} u \exp (4 \gamma t) .
\end{gathered}
$$

The generators associated with this subsubcase are

$$
\begin{gathered}
\mathbf{X}_{1}=\frac{1}{4 \gamma} \exp (4 \gamma t) \frac{\partial}{\partial t}-\frac{1}{4} u \exp (4 \gamma t) \frac{\partial}{\partial u}, \\
\mathbf{X}_{2}=\frac{\partial}{\partial t} .
\end{gathered}
$$

TABLE 5: Commutator table of the tumor equation.

\begin{tabular}{lcc}
\hline$\left[\mathbf{X}_{\mathrm{i}}, \mathbf{X}_{\mathrm{j}}\right]$ & $\mathbf{X}_{1}$ & $\mathbf{X}_{2}$ \\
\hline $\mathbf{X}_{1}$ & 0 & $-4 \gamma \mathbf{X}_{1}$ \\
$\mathbf{X}_{2}$ & $4 \gamma \mathbf{X}_{1}$ & 0 \\
\hline
\end{tabular}

TABLE 6: Commutator table of the tumor equation.

\begin{tabular}{lccc}
\hline$\left[\mathbf{X}_{\mathbf{i}}, \mathbf{X}_{\mathbf{j}}\right]$ & $\mathbf{X}_{1}$ & $\mathbf{X}_{2}$ & $\mathbf{X}_{3}$ \\
\hline $\mathbf{X}_{1}$ & 0 & 0 & $-4 \gamma \mathbf{X}_{1}$ \\
$X_{2}$ & 0 & 0 & 0 \\
$X_{3}$ & $4 \gamma \mathbf{X}_{1}$ & 0 & 0 \\
\hline
\end{tabular}

The commutation relation for these generators is given in Table 5.

Subsubcase (b.2) $\left(D(u)=((1 / 4) u)^{4}\right.$ and $\left.K(u)=\gamma\right)$. This subsubcase with (61) yields to $c_{4}=0$ and hence the infinitesimals are

$$
\begin{gathered}
\xi=c_{1} x, \quad \tau=\frac{c_{3}}{4 \gamma} \exp (4 \gamma t)+c_{5}, \\
\phi=\frac{1}{4} u\left(2 c_{1}-c_{3} \exp (4 \gamma t)\right) .
\end{gathered}
$$

The generators associated with this subsubcase are

$$
\begin{gathered}
\mathbf{X}_{1}=\frac{1}{4 \gamma} \exp (4 \gamma t) \frac{\partial}{\partial t}-\frac{1}{4} u \exp (4 \gamma t) \frac{\partial}{\partial u}, \\
\mathbf{X}_{2}=x \frac{\partial}{\partial x}+\frac{1}{2} u \frac{\partial}{\partial u}, \quad \mathbf{X}_{3}=\frac{\partial}{\partial t} .
\end{gathered}
$$

The commutation relation for these generators is given in Table 6.

Subsubcase (b.3) $\left(D(u)=((1 / 4) u)^{4}\right.$ and $\left.K(u)=\gamma\right)$. In accordance with this subsubcase and (61), we obtain $c_{4}=0$ and hence the infinitesimals are

$$
\begin{gathered}
\xi=0, \quad \tau=\frac{c_{3}}{4 \gamma} \exp (4 \gamma t)+c_{5}, \\
\phi=-\frac{1}{4} c_{3} u \exp (4 \gamma t) .
\end{gathered}
$$

The generators corresponding to this subsubcase are

$$
\begin{gathered}
\mathbf{X}_{\mathbf{1}}=\frac{1}{4 \gamma} \exp (4 \gamma t) \frac{\partial}{\partial t}-\frac{1}{4} u \exp (4 \gamma t) \frac{\partial}{\partial u}, \\
\mathbf{X}_{2}=\frac{\partial}{\partial t} .
\end{gathered}
$$

Remark 1. Case (III) represents the particular case of (I) and (II). So by similar manipulation as in the previous cases, we obtain the same symmetry generators.

\section{Some Reduction}

In this section, we present solutions of (4) via reductions. These reductions are obtained by the similarity variables obtained through symmetry generators. 


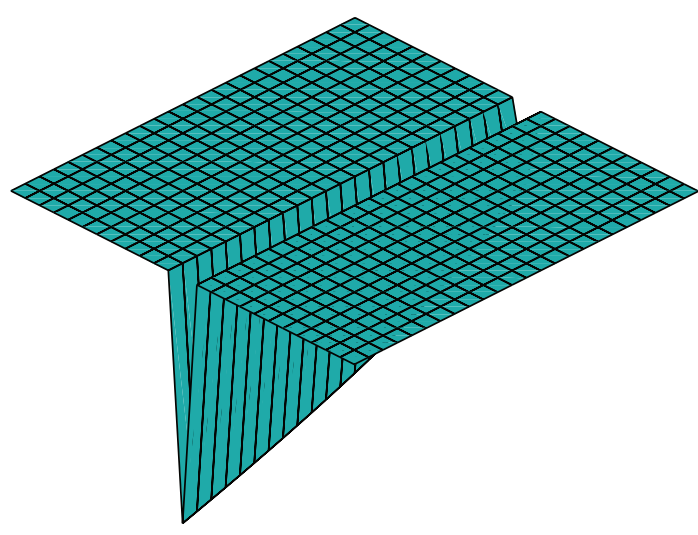

FIgURE 1: Plot of solution (74) with $c_{1}=1, c_{2}=0$.

Case $1(K(u)$ and $D(u)$ are arbitrary). In this case, we have only one generator; that is, $\mathbf{X}=\partial / \partial t$. Thus, the characteristic equation corresponding to this generator is

$$
\frac{d x}{0}=\frac{d t}{1}=\frac{d u}{0} .
$$

Solving the above equation it is straightforward [11] to find that it yields the similarity variables $r=x$ and $w=u$. Replacing $u$ in (4) in terms of new variables it becomes

$$
r D(w) w_{r r}+r D_{w} w_{r}^{2}+2 D(w) w_{r}-r K(w) w=0 .
$$

Case $2(K(u)=0$ and $D(u)$ are arbitrary). We consider the generator $\mathbf{X}_{1}=x(\partial / \partial x)+2 t(\partial / \partial t)$. Thus, the characteristic equation associated with this generator is

$$
\frac{d x}{x}=\frac{d t}{2 t}=\frac{d u}{0} .
$$

The similarity variables corresponding to the above equation become $r=x t^{-(1 / 2)}$ and $w=u$. These new variables reduce (4) to a ODE of the form

$$
r^{2} D(w) w_{r r}+r^{2} D_{w} w_{r}^{2}+2 r D(w) w_{r}+\frac{1}{2} r^{3} w_{r}=0 .
$$

Choosing $D(w)=1$ in the above equation, then the solution of the resulting equation is

$$
w(r)=c_{2}+c_{1}\left(-\frac{1}{r} \exp \left(\frac{-r^{2}}{4}\right)-\frac{1}{2} \sqrt{\Pi} \operatorname{Erf}\left(\frac{r}{2}\right)\right) .
$$

Recasting the above equation in its original coordinates, the exact solution of (4) becomes

$$
u(x, t)=c_{2}+c_{1}\left(-\frac{\sqrt{t}}{x} \exp \left(\frac{-x^{2}}{4 t}\right)-\frac{1}{2} \sqrt{\Pi} \operatorname{Erf}\left(\frac{x}{2 \sqrt{t}}\right)\right) .
$$

The graph of this solution is plotted in Figure 1.

Case $3\left(K(u)=\gamma+\lambda u^{1 / b}\right.$ and $\left.D(u)=a(b u)^{1 / b}\right)$. We take the generator

$$
\mathbf{X}_{1}=\frac{b}{\gamma} \exp \left(\frac{\gamma}{b} t\right) \frac{\partial}{\partial t}-b u \exp \left(\frac{\gamma}{b} t\right) \frac{\partial}{\partial u}
$$

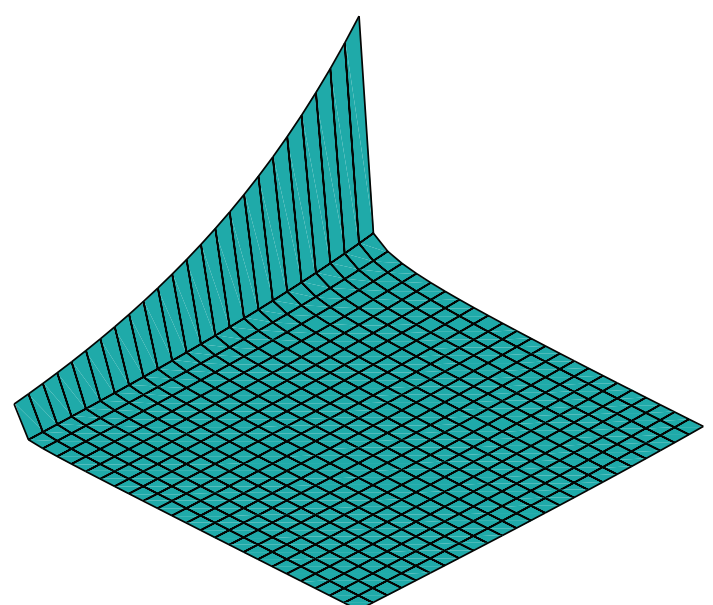

FIGURE 2: Plot of solution (79) with $c_{1}=0, \gamma=c_{2}=1$.

and its characteristic equation

$$
\frac{d x}{0}=\frac{\gamma}{b} \exp \left(\frac{-\gamma}{b} t\right) d t=\exp \left(\frac{-\gamma}{b} t\right) \frac{d u}{-b u}
$$

gives the similarity variables $r=x$ and $w=\exp (\gamma t) u$. In accordance of these similarities, (4) is transformed to the ODE in the form

$$
r w_{r r}+\frac{r}{b} \frac{1}{w} w_{r}^{2}+2 w_{r}-r \lambda b^{-(1 / b)} w=0 .
$$

Choosing $b=\lambda=\gamma=1$ in the above equation, then its solution becomes

$$
w(r)=\frac{c_{2}}{\sqrt{r}} \sqrt{\cosh \left(\sqrt{2} r+i c_{1}\right)} .
$$

Recasting the above equation in its original coordinates, the exact solution of (4) becomes

$$
u(x, t)=\exp (-t) \frac{c_{2}}{\sqrt{x}} \sqrt{\cosh \left(\sqrt{2} x+i c_{1}\right)} .
$$

The graph of this solution is plotted in Figure 2.

Case $4\left(K(u)=\gamma\right.$ and $\left.D(u)=a(b u)^{1 / b}\right)$. In this case, we consider the generator

$$
\mathbf{X}_{1}=\frac{b}{\gamma} \exp \left(\frac{\gamma}{b} t\right) \frac{\partial}{\partial t}-b u \exp \left(\frac{\gamma}{b} t\right) \frac{\partial}{\partial u} .
$$

The characteristic equation corresponding to this generator is

$$
\frac{d x}{0}=\frac{\gamma}{b} \exp \left(\frac{-\gamma}{b} t\right) d t=\exp \left(\frac{-\gamma}{b} t\right) \frac{d u}{-b u} ;
$$

solving the above characteristic equation gives $r=x$ with $w=\exp (\gamma t) u$. These variables can be used to recast (4) to an ODE,

$$
r w_{r r}+\frac{r}{b} \frac{1}{w} w_{r}^{2}+2 w_{r}=0
$$




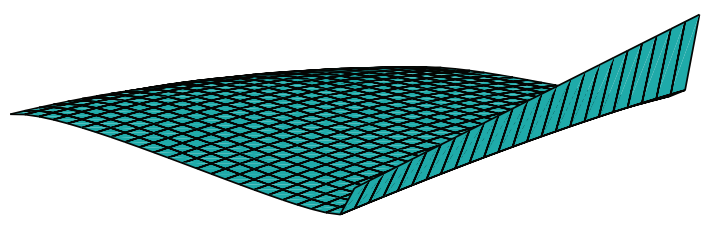

Figure 3: Plot of solution (84) with $b=1 / 4, \gamma=c_{1}=c_{2}=1$.

Choosing $b=(1 / 4)$ in $(82)$, then its solution becomes

$$
w(r)=\left(\frac{-r}{5\left(c_{1}-c_{2} r\right)}\right)^{-1 / 5} .
$$

Recasting the above equation in its original coordinates, the exact solution of (4) becomes

$$
u(x, t)=\exp (-\gamma t)\left(\frac{-x}{5\left(c_{1}-c_{2} x\right)}\right)^{-1 / 5} .
$$

The graph of this solution is plotted in Figure 3.

\section{Conflict of Interests}

The authors declare that there is no conflict of interests regarding the publication of this paper.

\section{Acknowledgment}

The authors would like to acknowledge the support provided by the Deanship of Research at King Fahd University of Petroleum and Minerals (KFUPM) for funding this work through Project IN111008.

\section{References}

[1] D. S. Jones, M. Plank, and B. D. Saleem, Differential Equations and Mathematical Biology, Mathematical \& Computational Biology, Chapman and Hall/CRC, New York, NY, USA, 2011.

[2] G. C. Cruywagen, D. E. Woodward, P. Tracqui, G. T. Bartoo, J. D. Murray, and C. A. Ellsworth, "The modelling of diffusive tumours," Journal of Biological Systems, vol. 3, no. 4, pp. 937-945, 1995.

[3] S. Moyo and P. G. L. Leach, "Symmetry methods applied to a mathematical model of a tumour of the brain," Proceedings of Institute of Mathematics of NAS of Ukraine, vol. 50, pp. 204-210, 2004.

[4] P. Tracqui, G. C. Cruywagen, D. E. Woodward, G. T. Bartoo, J. D. Murray, and E. C. Alvord, "A mathematical model of glioma growth: the effect of chemotherapy on spatio-temporal growth," Cell Proliferation, vol. 28, no. 1, pp. 17-31, 1995.

[5] A. H. Bokhari, A. H. Kara, and F. D. Zaman, "On the solutions and conservation laws of the model for tumor growth in the brain," Journal of Mathematical Analysis and Applications, vol. 350, no. 1, pp. 256-261, 2009.

[6] S. Lie, Theorie der Transoformationsgruppen, vol. 3, B. G. Tubner, Ed., Liepzig, Germany, 1893.

[7] G. W. Bluman and S. C. Anco, Symmetry and Integration Methods for Differential Equations, Springer, New York, NY, USA, 2002.
[8] G. W. Bluman, A. F. Cheviakov, and S. C. Anco, Applications of Symmetry Methods to Partial Differential Equations, Springer, New York, NY, USA, 2010.

[9] G. W. Bluman and S. Kumei, Symmetries and Differential Equations, Springer, New York, NY, USA, 1989.

[10] B. J. Cantwell, Introduction to Symmetry Analysis, Cambridge University Press, Cambridge, UK, 2002.

[11] A. Ahmad, A. H. Bokhari, A. H. Kara, and F. D. Zaman, "Symmetry classifications and reductions of some classes of $(2+$ 1)-nonlinear heat equation," Journal of Mathematical Analysis and Applications, vol. 339, no. 1, pp. 175-181, 2008. 


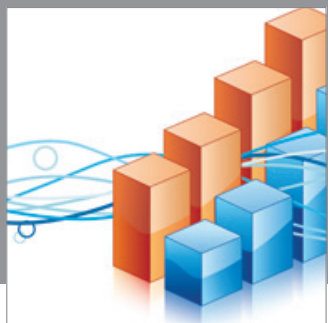

Advances in

Operations Research

mansans

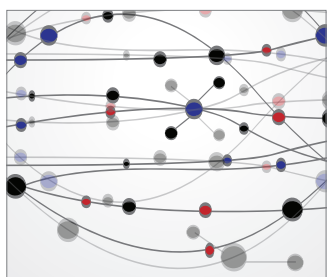

The Scientific World Journal
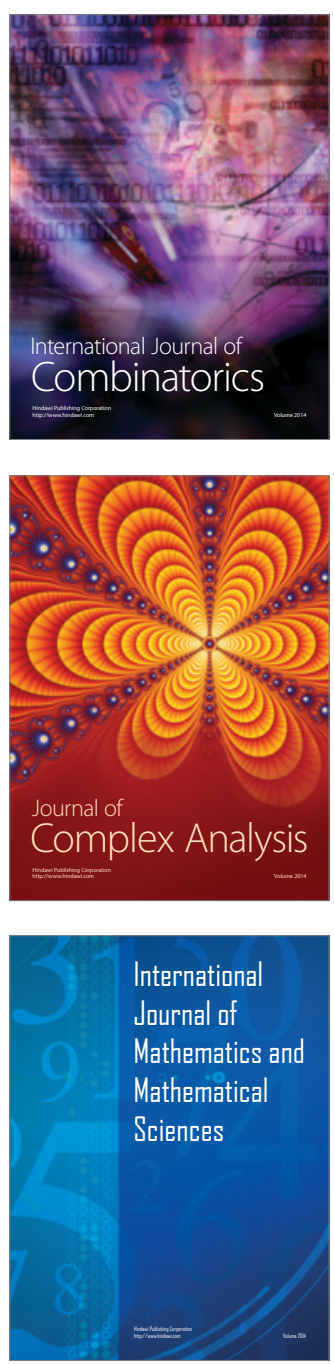
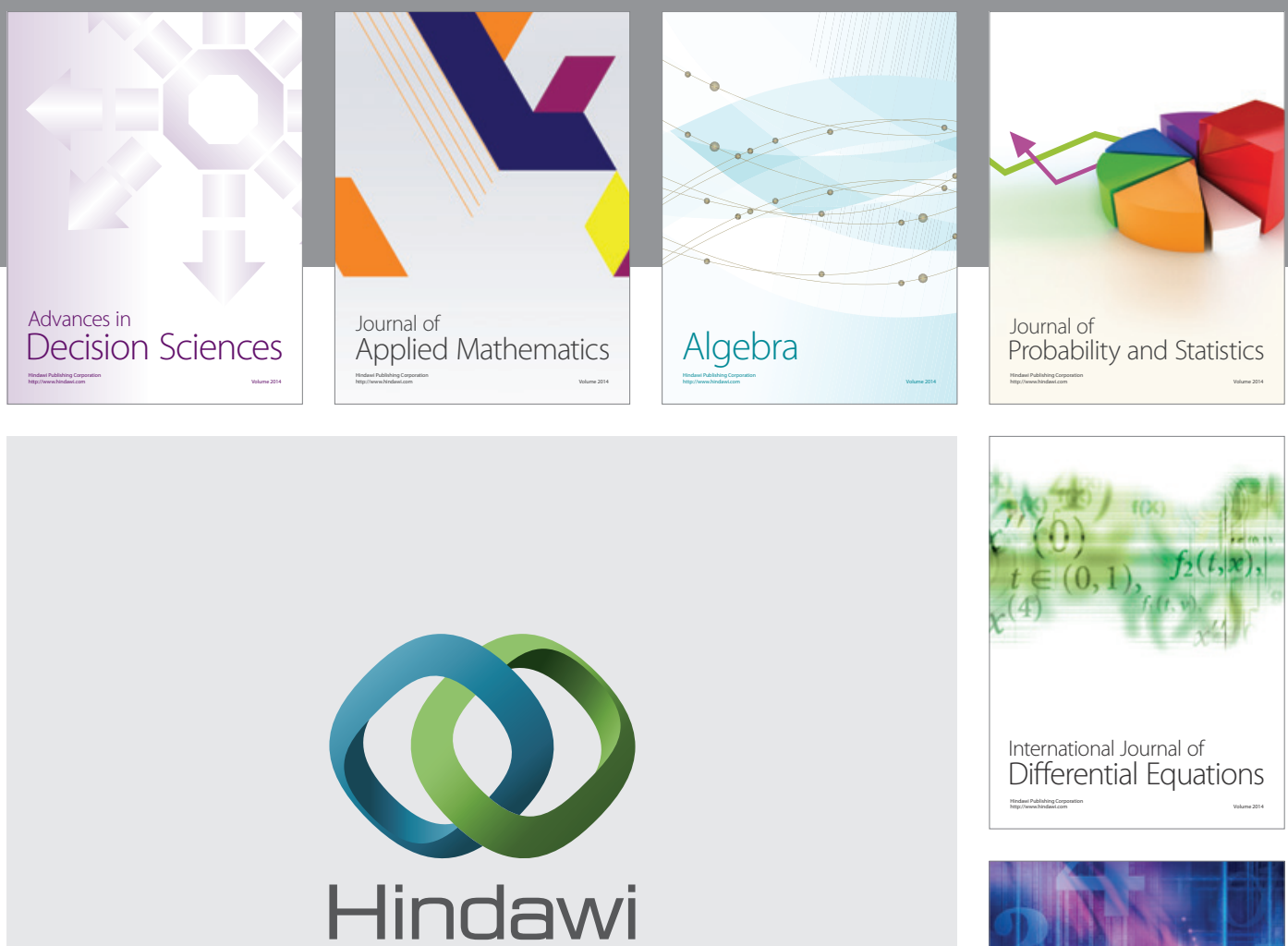

Submit your manuscripts at http://www.hindawi.com
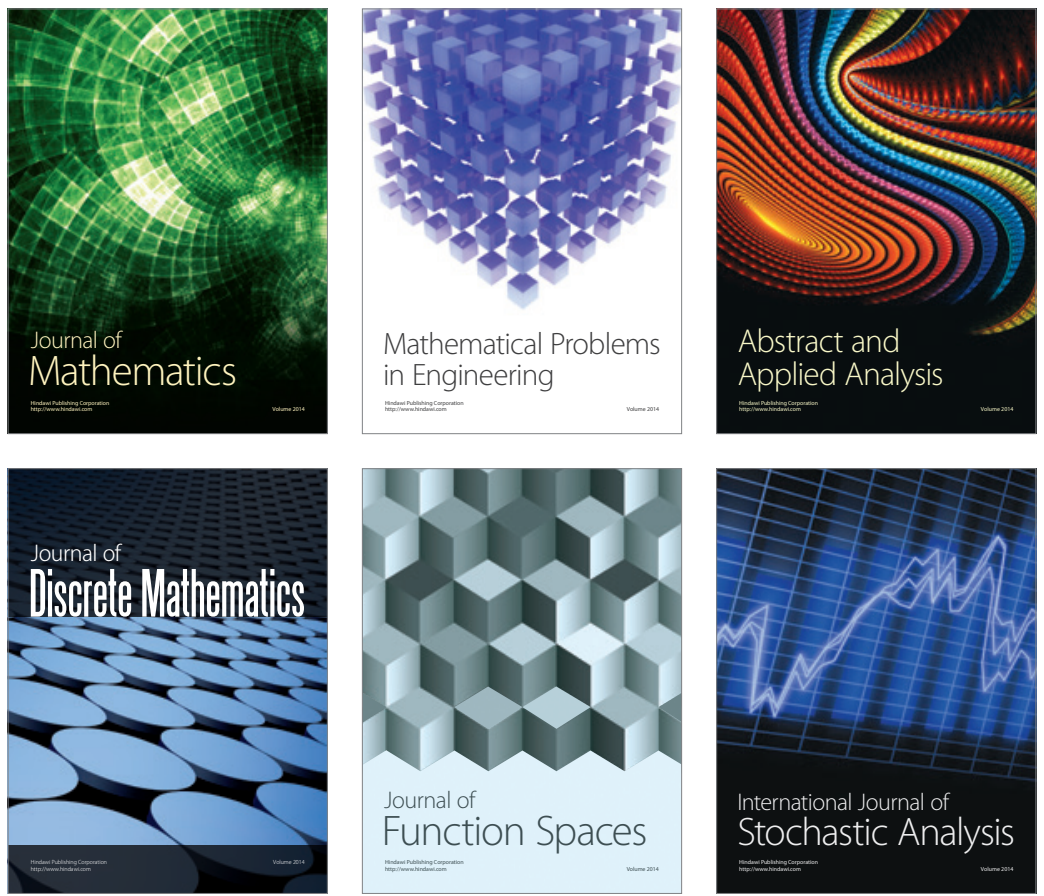

Journal of

Function Spaces

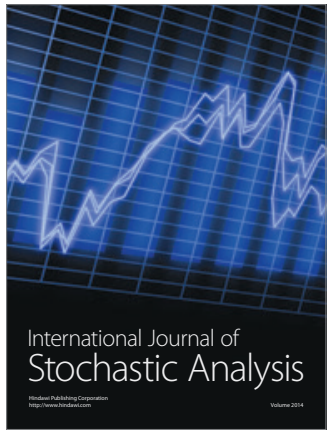

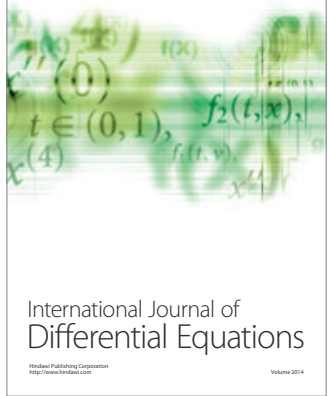
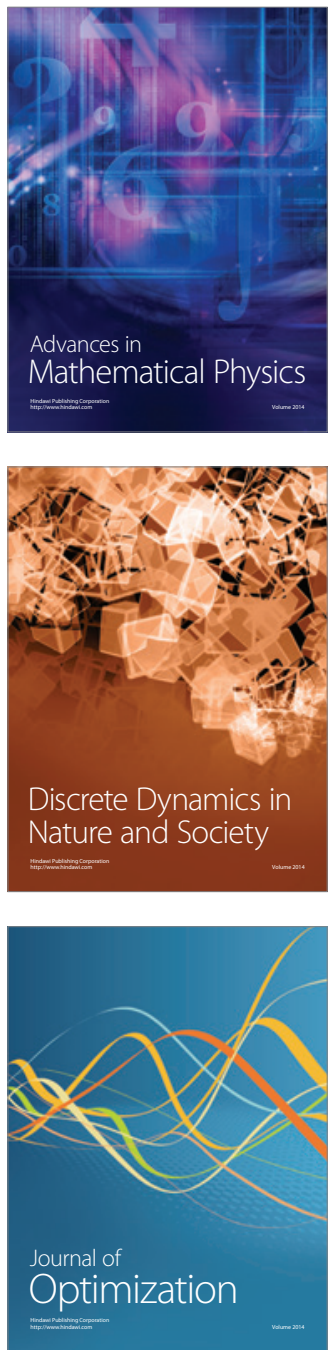